\title{
CRITICAL VALUES LIE ON A LINE
}

AZAT AINOULINE

Received 9 September 2003

We prove that critical values set of a differentiable map lies on a line of certain smoothness class.

\section{Introduction}

For those familiar with the "space-filling curves" topic, the headline of the paper is no surprise. G. Peano in 1890 constructed the first such continuous function $f_{p}:[0,1] \stackrel{\text { onto }}{\longrightarrow}$ $[0,1]^{2}$. Nowadays, the topic is well developed by a number of mathematicians (see [9]).

A further question is how smooth can the line be? Or how far from rectifiable is the line? In 1935, Whitney [10] published his example of a $C^{1}$-function $f_{W}:[0,1]^{2} \stackrel{\text { onto }}{\longrightarrow}[0,1]$ not constant on a connected set of critical points. The author in [2] constructed Whitneytype examples of maps $f \in C^{k}\left(\mathbb{R}^{n}, \mathbb{R}^{m}\right)$ for maximal possible $k$.

Theorem $1.1[2]$. For any $n, m \in \mathbb{N}$, there exist a map $f:[0,1]^{n} \rightarrow[0,1]^{m}$, contained in $C^{k}$ for all real $k<n / m$, and a connected set $E \subseteq[0,1]^{n}$ such that every partial derivative of $f$ of order $<n / m$ vanishes on $E$ and $f(E)=[0,1]^{m}$.

Theorem 1.2 [2]. Let $n, m, p$ be nonnegative integer numbers, $n>m>p$; then there exists a map $f: \mathbb{R}^{n} \rightarrow \mathbb{R}^{m}$, contained in $C^{k}$ for all real $k<(n-p) /(m-p)$, and a connected subset $E$ of points of rank $p$ such that $f(E)$ contains an open set.

The first theorem holds important information that $[0,1]^{m}$ can be covered by a line of smoothness class $C^{<1 / m}$ (i.e., we write $f \in C^{<k_{0}}$ if $f \in C^{k}$ for every $k<k_{0}$ ). In this paper, the author determines the smoothness class of a line that can cover a critical values set of a differentiable map.

Main theorem 1. Let $F: \mathbb{R}^{n} \stackrel{C^{k \cdot \lambda}}{\longrightarrow} \mathbb{R}^{m}, k \in \mathbb{N}, \lambda \in[0,1)$; then $F\left(C_{p}(F)\right) \subseteq f\left(\Sigma_{\mu} f\right)$ for some $C^{<\mu}$ - function $f: \mathbb{R} \rightarrow \mathbb{R}^{m}$, where $\mu=\max \{1 /(p+((n-p) /(k+\lambda))), 1 / m\}$ and $\Sigma_{\mu} f:=$ $\{x \in \mathbb{R}$ : any partial derivative of $f$ of order $<\mu$ vanishes at $x\}$. 
This is a Sard-type theorem, and sharpness of the $\mu$ can be seen in the results, where necessary and sufficient conditions for the Morse-Sard theorem are establi shed, that are in $[11,3]$ for the case $C^{k}\left(\mathbb{R}^{1}, \mathbb{R}^{1}\right)$, and [1] for the case $C^{k}\left(\mathbb{R}^{n}, \mathbb{R}^{1}\right)$.

\section{Notations and preliminary lemmas}

Definition 2.1. Let $f: \mathbb{R}^{m} \rightarrow \mathbb{R}^{n}$ be a continuous function and $\lambda \in[0,1)$. It is said that $f \in C^{0 \cdot \lambda}$ if $f$ satisfies a $\lambda$-Hölder condition: for every compact neighborhood $U$, there exists $M>0$ such that

$$
|f(x)-f(y)| \leqslant M \cdot|x-y|^{\lambda} \quad \forall x, y \in U \text {. }
$$

Definition 2.2. For $k \in \mathbb{N}, \lambda \in\left[0,1\right.$ ), a function $f: \mathbb{R}^{m} \rightarrow \mathbb{R}^{n}$ is a $C^{k \cdot \lambda}$-function (or $f \in C^{k \cdot \lambda}$ ) if $f \in C^{k}$ and every $k$ th partial derivative of $f$ is a $C^{0 \cdot \lambda}$-function. If $f \in C^{p \cdot \beta}$ for all $p+\beta<k+\lambda, f \in C^{k \cdot \lambda}$.

Definition 2.3. For $f: \mathbb{R}^{m} \stackrel{C^{0 \cdot \lambda}}{\longrightarrow} \mathbb{R}^{n}$, define partial derivatives of order $\lambda: f_{1}^{(\lambda)}, \ldots, f_{m}^{(\lambda)}$ by the formula

$$
f_{i}^{(\lambda)}(a)=\lim _{t \rightarrow 0} \operatorname{sign}(t) \frac{f\left(a_{1}, \ldots, a_{i-1}, a_{i}+t, a_{i+1}, \ldots, a_{n}\right)-f(a)}{|t|^{\lambda}}
$$

for $a=\left(a_{1}, \ldots, a_{m}\right) \in \mathbb{R}^{m}$. If all partial derivatives of order $\lambda$ are continuous, $f \in C^{\lambda}$.

Definition 2.4. For $k \in \mathbb{R}^{+}$, a function $f: \mathbb{R}^{m} \stackrel{C^{[k] \cdot k-[k]}}{\longrightarrow} \mathbb{R}^{n}$ is a $C^{k}$-function (or $f \in C^{k}$ ) if $f \in C^{[k]}$ and every [k]th partial derivative of $f$ is a $C^{k-[k]}$-function, where $[k]$ is the integer part of $k$. If $f \in C^{k}$ for every $k<k_{0}, f \in C^{<k_{0}}$.

We begin by setting $K_{0}^{n}=\left\{Q_{i_{0}}, i_{0} \in \mathbb{N}\right\}$, where $Q_{i_{0}}$ is a closed cube in $\mathbb{R}^{n}$ with side length 1 and every coordinate of any vertex of $Q_{i_{0}}$ is an integer. In general, having constructed the cubes of $K_{s-1}^{n}$, divide each $Q_{i_{0}, i_{1}, i_{2}, \ldots, i_{s-1}} \in K_{s-1}^{n}$ into $2^{n}$ closed cubes of side $1 / 2^{s}$, and let $K_{s}^{n}$ be the set of all these cubes. More precisely, we will write

$$
K_{s}^{n}=\left\{Q_{i_{0}, i_{1}, i_{2}, \ldots, i_{s-1}, i_{s}} ; Q_{i_{0}, i_{1}, i_{2}, \ldots, i_{s-1}, i_{s}} \subseteq Q_{i_{0}, i_{1}, i_{2}, \ldots, i_{s-1}} \in K_{s-1}^{n}, 1 \leqslant i_{s} \leqslant 2^{n}\right\} .
$$

We also define

(i) $K^{n}=\bigcup_{s+1 \in \mathbb{N}} K_{s}^{n}$ (note that $K^{n}$ is defined for $\mathbb{R}^{n}$ );

(ii) $S(\delta)$-the length of a side of $\delta \in K^{n}$.

Lemma 2.5. Let $E_{1}, E_{2}$ be copies of $\mathbb{R}$. For all $n, m \in \mathbb{N}$, there exists continuous $H_{n, m}$ : $[0,1] \stackrel{\text { onto }}{\longrightarrow}[0,1]^{2} \subseteq E_{1} \times E_{2}$ such that

(1) if $\alpha \in K_{(n+m) \cdot s}^{1}$, then $H_{n, m}(\alpha)=\alpha^{\prime} \times \alpha^{\prime \prime}$, where $\alpha^{\prime} \in K_{n \cdot s}^{1}, \alpha^{\prime \prime} \in K_{m \cdot s}^{1}, \alpha^{\prime} \in E_{1}$, and $\alpha^{\prime \prime} \in E_{2}$,

(2) if $\alpha^{\prime} \times \alpha^{\prime \prime} \subseteq[0,1]^{2}$ such that $\alpha^{\prime} \subseteq E_{1}, \alpha^{\prime \prime} \subseteq E_{2}, \alpha^{\prime} \in K_{n \cdot s}^{1}$, and $\alpha^{\prime \prime} \in K_{m \cdot s}^{1}$, then $H_{n, m}^{-1}\left(\operatorname{int}\left(\alpha^{\prime} \times \alpha^{\prime \prime}\right)\right) \subseteq \alpha \in K_{(n+m) \cdot s^{*}}^{1}$

Proof. We define for every $n, m \in \mathbb{N}$ a space-filling function $H_{n, m}$ as follows. 


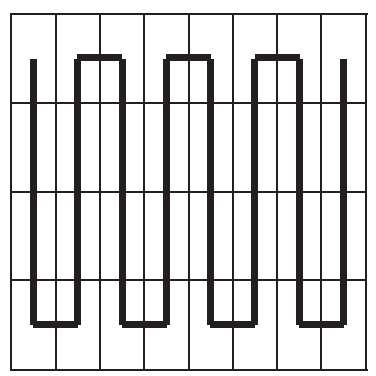

Figure 2.1. HU.

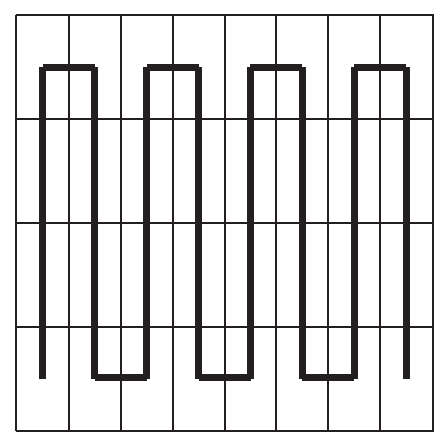

Figure 2.2. HD.

If the interval $[0,1]$ can be mapped continuously onto the square $[0,1]^{2}$, then after partitioning $[0,1]$ into $2^{n+m}$ congruent subintervals and $[0,1]^{2}$ into $2^{n+m}$ congruent subrectangles with sides $1 / 2^{n}, 1 / 2^{m}$, each subinterval can be mapped continuously onto one of the subrectangles.

Next, each subinterval is, in turn, partitioned into $2^{n+m}$ congruent subintervals, and each subrectangle into $2^{n+m}$ congruent subrectangles with sides $1 / 2^{2 n}, 1 / 2^{2 m}$ and the argument is repeated. If this is carried on indefinitely, $[0,1]$ and $[0,1]^{2}$ are partitioned into $2^{(n+m) s}$ congruent replicas, each with sides $1 / 2^{n s}, 1 / 2^{m s}$ for $s \in \mathbb{N}$.

We need to demonstrate that the subsquares can be arranged so that adjacent subintervals correspond to adjacent subsquares with an edge in common, and so that the inclusion relationships are presented, that is, if a rectangle corresponds to an interval, then its subrectangles correspond to the subintervals of that interval.

We will use here combination of four different methods to construct these space-filling curves. These methods are based on an idea of Peano [9]. For future use, we designate them as $\operatorname{VL}(n, m), \operatorname{VR}(n, m), \operatorname{HD}(n, m), \mathrm{HU}(n, m)$.

If we have a rectangle, then using any of those methods gives us $2^{n+m}$ equal subrectangles which are ordered according to the order assigned by the method used.

Figures 2.1, 2.2, 2.3, and 2.4 give us a basic idea of how these four methods work. 


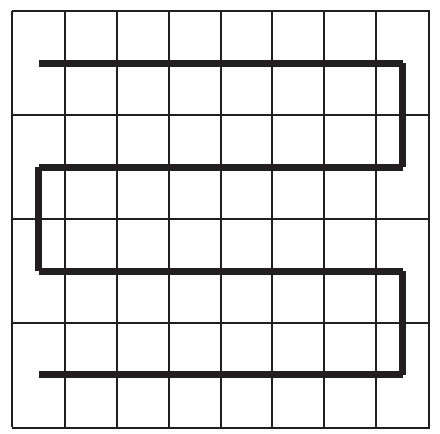

Figure 2.3. VL.

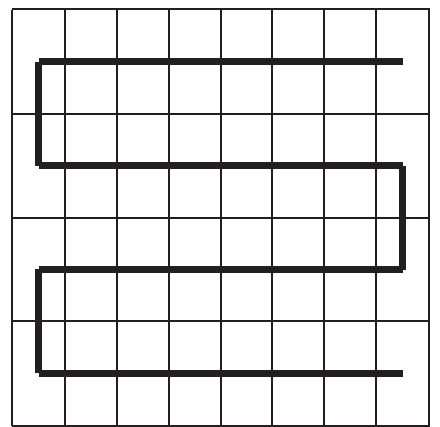

Figure 2.4. VR.

Note. As soon as the curves in all four methods are passing through all the subrectangles, the only essential difference among the four methods is the disposition of start and end points. That is denoted in abbreviations of the methods: V-vertical, H-horizontal, L-left, R-right, U-up, D-down.

Further, to create the next iteration curve, we will give the means of how to present each of the subrectangles from the previous iteration (see Figures 2.5, 2.6, 2.7, and 2.8).

Finally, in Figures 2.9 and 2.10, we indicate how this process is to be carried out for the next iteration.

Now we can define $H_{n, m}$ for any $n, m \in \mathbb{N}$.

Definition 2.6. Every $t \in[0,1]$ is uniquely determined by a sequence of nested closed intervals (that are generated by our successive partitioning), the lengths of which shrink to 0 . With this sequence, there corresponds a unique sequence of nested closed squares, the diagonals of which shrink into a point, and which define a unique point in $[0,1]^{2}$, the image $H_{n, m}(t)$ of $t$.

The function $H_{n, m}$ satisfies the properties (1), (2) of Lemma 2.5 by its definition. 


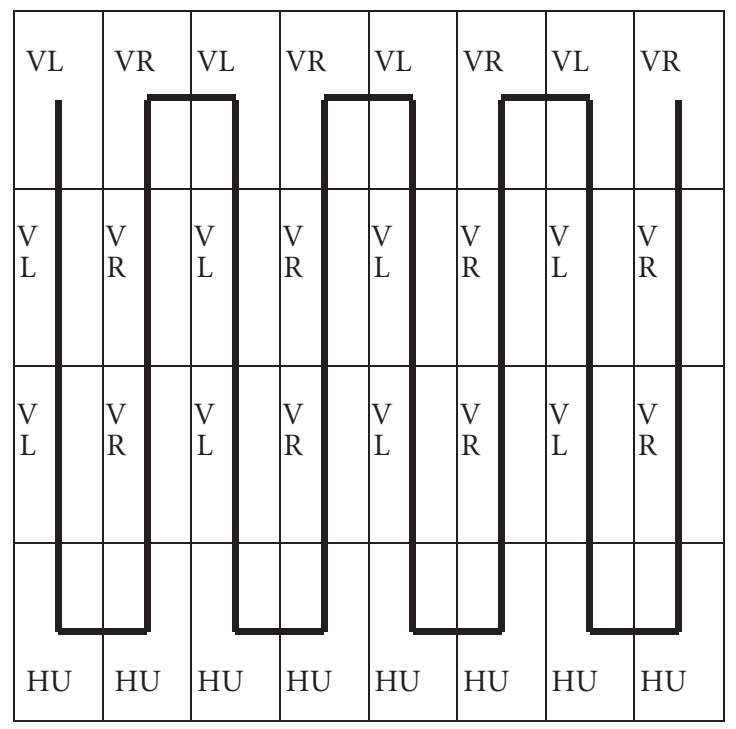

Figure 2.5. HU.

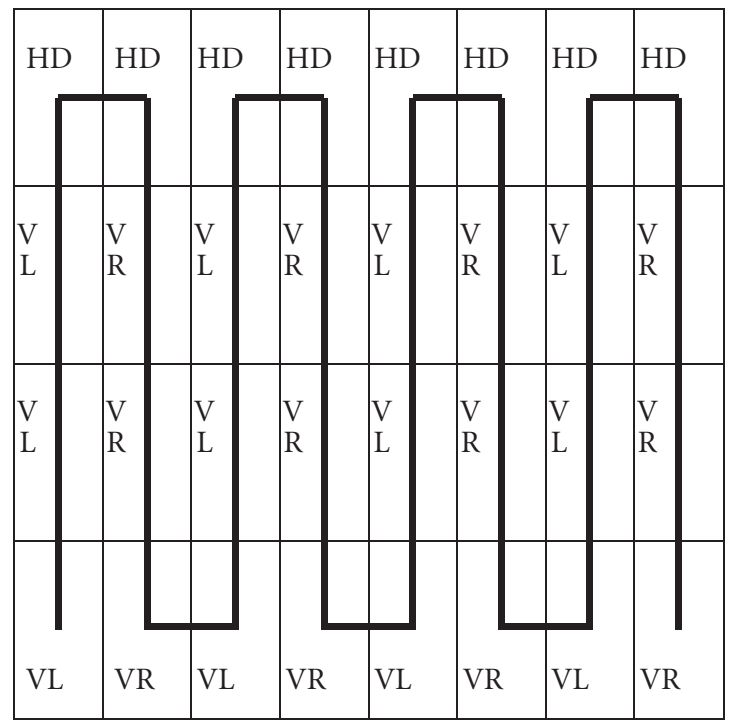

Figure 2.6. HD.

Lemma 2.7. Let $E_{1}, E_{2}$ be copies of $\mathbb{R}$. For all $\tilde{n}, \tilde{m}: \mathbb{N} \rightarrow \mathbb{N}$, there exists continuous function $H_{\tilde{n}, \tilde{m}}:[0,1] \stackrel{\text { onto }}{\longrightarrow}[0,1]^{2} \subseteq E_{1} \times E_{2}$ such that

(1) if $\alpha \in K_{\sum_{i=1}^{s} \tilde{n}(i)+\tilde{m}(i)}^{1}$ for some $s \in \mathbb{N}$, then $H_{\tilde{n}, \tilde{m}}(\alpha)=\alpha^{\prime} \times \alpha^{\prime \prime}$, where $\alpha^{\prime} \in K_{\sum_{i=1}^{s} \tilde{n}(i)}^{1}$, $\alpha^{\prime \prime} \in K_{\sum_{i=1}^{s} \tilde{m}(i)}^{1}$ 


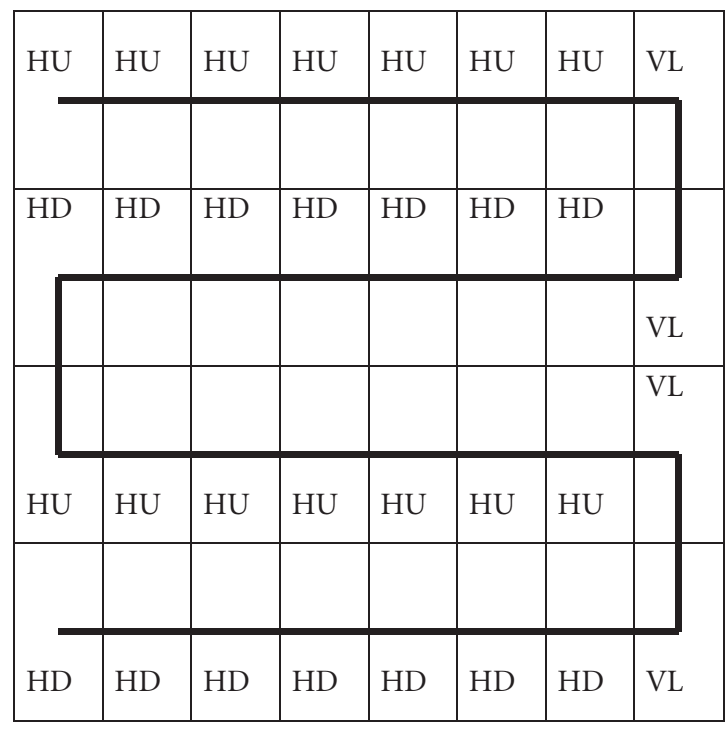

Figure 2.7. VL.

\begin{tabular}{|l|l|l|l|l|l|l|l|}
\hline VR & HU & HU & HU & HU & HU & HU & HU \\
\hline & & & & & & & \\
\hline & HD & HD & HD & HD & HD & HD & HD \\
\hline VR & & & & & & & \\
\hline VR & & & & & & & \\
\hline VR & HD & HD & HD & HD & HD & HD & HD \\
\hline
\end{tabular}

Figure 2.8. VR.

(2) if $\alpha^{\prime} \times \alpha^{\prime \prime} \subseteq[0,1]^{2}$ such that $\alpha^{\prime} \subseteq E_{1}, \alpha^{\prime \prime} \subseteq E_{2}, \alpha^{\prime} \in K_{\sum_{i=1}^{s} \tilde{n}(i)}^{1}$, and $\alpha^{\prime \prime} \in K_{\sum_{i=1}^{s} \tilde{m}(i)}^{1}$ for some $s \in \mathbb{N}$, then $H_{\tilde{n}, \tilde{m}}^{-1}\left(\operatorname{int}\left(\alpha^{\prime} \times \alpha^{\prime \prime}\right)\right) \subseteq \alpha \in K_{\sum_{i=1}^{s} \tilde{n}(i)+\tilde{m}(i)}^{1}$.

Proof. The proof is similar to the proof of Lemma 2.5, with the only difference that if we used, for instance, a method $\operatorname{VL}(n, m)$ to decompose a subrectangle on an iteration $s$ in 


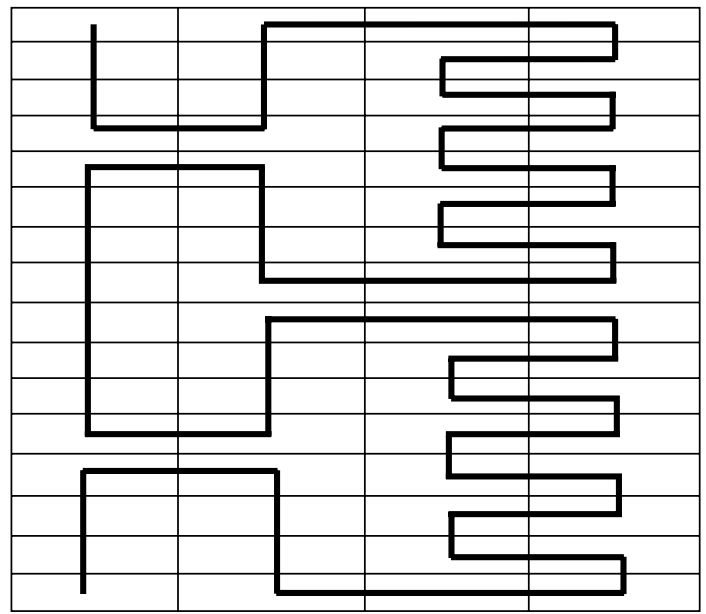

Figure 2.9. Next iteration when started with method VL.

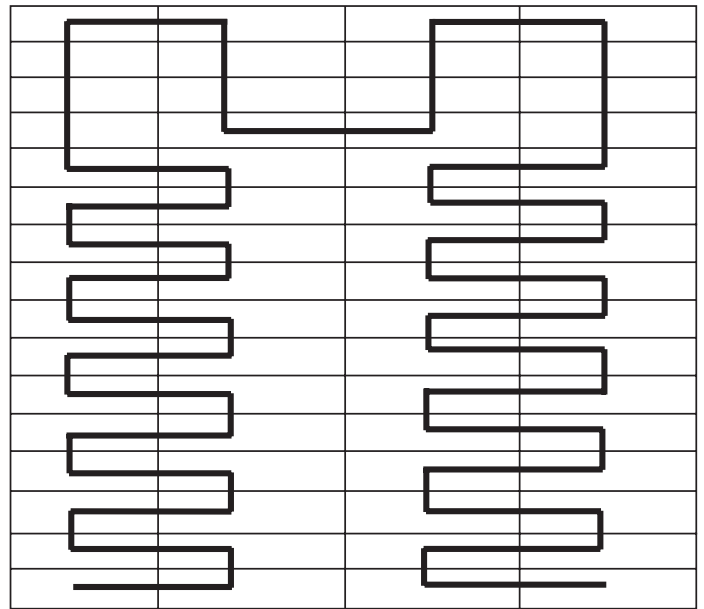

Figure 2.10. Next iteration when started with method HD.

Lemma 2.5, then here we use a corresponding method $\operatorname{VL}(n(s), m(s))$ on the iteration $s$.

Definition $2.8[2]$. Call a function $f_{n}:[0,1] \rightarrow[0,1]^{n}$ cubes-preserving if it has the following properties:

(i) if $\alpha \subseteq[0,1]$ and for some $s \in \mathbb{N}, \alpha \in K_{n \cdot s}^{1}$ implies $f_{n}(\alpha) \subseteq \delta$ for some $\delta \in K_{s}^{n}$,

(ii) if $\delta \subseteq[0,1]^{n}$ and for some $s \in \mathbb{N}, \delta \in K_{s}^{n}$ implies $f_{n}^{-1}(\operatorname{int}(\delta)) \subseteq \alpha$ for some $\alpha \in$ $K_{n \cdot s}^{1}$,

where $\operatorname{int}(\delta)$ is the set of interior points of $\delta$. 
Note that a continuous cubes-preserving function $f_{n}$ is a space-filling and measurepreserving function, that is, with the property that if $\alpha \subseteq[0,1]$ and for some $s \in \mathbb{N}, \alpha \in$ $K_{n \cdot s}^{1}$, then $f_{n}(\alpha)=\delta$ for some $\delta \in K_{s}^{n}$.

Theorem 2.9 (space-filling function) [2, Theorem 1]. For every $n \in \mathbb{N}$, there exists a continuous cubes-preserving function

$$
f_{n}:[0,1] \stackrel{\text { onto }}{\longrightarrow}[0,1]^{n}
$$

Definition 2.10. For $m, n \in \mathbb{N}, k \in \mathbb{R}$, a function $\psi: B \subseteq \mathbb{R}^{m} \rightarrow \mathbb{R}^{n}$ is $D^{k}$-function if there exist $K>0$ such that for all $b, b^{\prime} \in B$

$$
\left|\psi(b)-\psi\left(b^{\prime}\right)\right|^{k} \leqslant K\left|b-b^{\prime}\right|
$$

\subsection{Properties of $D^{k}$-functions}

Extension on closure property [1]. If $f: A \subseteq \mathbb{R}^{m} \stackrel{D^{k}}{\longrightarrow} \mathbb{R}^{n}$ for some $k>0$, and $\bar{A}$ is the closure of $A$, then there exists a unique function $\bar{f}: \bar{A} \subseteq \mathbb{R}^{m} \stackrel{C^{0}}{\longrightarrow} \mathbb{R}^{n}$ such that $\bar{f} \uparrow A=f$, and $\bar{f}$ is a $D^{k}$-function.

Composition property [1]. If $g \in D^{k}$ and $f \in D^{p}$, then $g \circ f \in D^{k p}$.

Subsets property [1]. If $f: A \subseteq \mathbb{R}^{m} \stackrel{D^{k}}{\longrightarrow} \mathbb{R}^{m}$ for some $k>0$, then $f \uparrow B \in D^{k}$ for any $B \subseteq A$. $C^{<k}$-extension on $\mathbb{R}$ property. If $F: B \subseteq \mathbb{R} \stackrel{D^{1 / k}}{\longrightarrow} \mathbb{R}^{m}, k>0$, then $F=f \uparrow B$ for some function $f: \mathbb{R} \stackrel{C^{<k}}{\longrightarrow} \mathbb{R}^{m}$, with range $(F) \subseteq f\left(\Sigma_{k} f\right)$.

We prove this property as follows. Let $f \uparrow \bar{B}$ be the $D^{1 / k}$ extension of the function $F$ on the closed set $\bar{B}$ the closure of $B$, that exists and is unique by the "extension on closure property." Then let $T$ be a real number such that

$$
\forall b, b^{\prime} \in \bar{B} \quad\left|f(b)-f\left(b^{\prime}\right)\right|^{1 / k} \leqslant T\left|b-b^{\prime}\right|
$$

and let $A=\operatorname{range}(f \uparrow \bar{B})$; then $\operatorname{range}(F) \subseteq A$.

We now define the function $f: \mathbb{R} \rightarrow \mathbb{R}^{m}$ as follows: if there exists a point $\bar{b} \in \mathbb{R}$ such that $\bar{b}=\max \{b \in \bar{B}\}$, then for all $x \geqslant \bar{b}, f(x)=f(\bar{b})$, respectively, if there exists a point $\underline{b} \in \mathbb{R}$ such that $\underline{b}=\min \{b \in \bar{B}\}$; then for all $x \leqslant \underline{b}, f(x)=f(\underline{b})$.

We designate $Z(\bar{B})=\left\{\left(b, b^{\prime}\right) \subseteq \mathbb{R} \backslash \bar{B} ; b<b^{\prime}, b, b^{\prime} \in \bar{B}\right\}$; this set is countable and we can write $Z(\bar{B})=\left\{\left(b_{n}, b_{n}^{\prime}\right) ; n \in \mathbb{N}\right\}$, where $b_{n}, b_{n}^{\prime} \in \bar{B}$.

Let $f=\left(f_{1}, \ldots, f_{i}, \ldots, f_{m}\right)$, where $f_{i}: \bar{B} \rightarrow \mathbb{R}, 1 \leqslant i \leqslant m$, are the component functions of the function $f \uparrow \bar{B}$; then for all $n \in \mathbb{N}$, for all $x \in\left(b_{n}, b_{n}^{\prime}\right)$, and for all $i(1 \leqslant i \leqslant m)$, we define

$$
f_{i}(x)=\left(f_{i}\left(b_{n}^{\prime}\right)-f_{i}\left(b_{n}\right)\right) \cdot g\left(\frac{x-b_{n}}{b_{n}^{\prime}-b_{n}}\right)+f_{i}\left(b_{n}\right),
$$


where (following $[6$, page 6$]) g: \mathbb{R}^{1} \rightarrow[0,1]$ is a smooth map such that

$$
\begin{gathered}
g \uparrow(-\infty, 0]=0, \\
g \uparrow[1, \infty)=1, \\
g^{\prime}(x)>0 \quad \text { for } 0<x<1 .
\end{gathered}
$$

Then $f$ is defined for all $x \in \mathbb{R}$, continuous, smooth on $\mathbb{R} \backslash \bar{B}$ and $A \subseteq \operatorname{range}(f)$. To finish the proof of $C^{<k}$-extension on $\mathbb{R}$ property, it suffices to show that

$$
f_{i}^{(t)}\lceil\bar{B}=0 \quad \forall i(1 \leqslant i \leqslant m), \forall t \in\{1,2, \ldots,[k]\} \cup([k], k) .
$$

It is evident for nonlimit points of $\bar{B}$. Let $B^{\prime} \subseteq \bar{B}$ be the set of limit points of $\bar{B}$.

Case 1. If $k \leqslant 1$, then for all $b \in B^{\prime}$ and some fixed $t: 0 \leqslant t<k$,

$$
\left|f_{i}^{(t)}(b)\right|=\lim _{h \rightarrow 0} \frac{\left|f_{i}(b+h)-f_{i}(b)\right|}{|h|^{t}}
$$

Note that we may suppose without loss of generality that

$$
\begin{gathered}
h>0, \\
b+h \in\left(b_{n}, b_{n}^{\prime}\right) \quad \text { for some } n \in \mathbb{N}, \\
\Delta_{n}:=b+h-b_{n} .
\end{gathered}
$$

Then

$$
\begin{aligned}
\frac{\left|f_{i}(b+h)-f_{i}(b)\right|}{|h|^{t}} & \leqslant \frac{\left|f_{i}\left(b_{n}\right)-f_{i}(b)\right|+\left|f_{i}(b+h)-f_{i}\left(b_{n}\right)\right|}{\left(\left|b_{n}-b\right|+\Delta_{n}\right)^{t}} \\
& \leqslant \frac{\left|f_{i}\left(b_{n}\right)-f_{i}(b)\right|}{\left(\left|b_{n}-b\right|+\Delta_{n}\right)^{t}}+\frac{\left|f_{i}(b+h)-f_{i}\left(b_{n}\right)\right|}{\left(\left|b_{n}-b\right|+\Delta_{n}\right)^{t}} \\
& \leqslant \frac{T^{k}\left|b_{n}-b\right|^{k}}{\left(\left|b_{n}-b\right|\right)^{t}}+\frac{\left|f_{i}(b+h)-f_{i}\left(b_{n}\right)\right|}{\Delta_{n}^{t}} .
\end{aligned}
$$

We consider each summand of (2.12) separately:

$$
\frac{T^{k}\left|b_{n}-b\right|^{k}}{\left(\left|b_{n}-b\right|\right)^{t}}=T^{k}\left|b_{n}-b\right|^{k-t}
$$

where $k-t>0$;

$$
\begin{aligned}
\frac{\left|f_{i}(b+h)-f_{i}\left(b_{n}\right)\right|}{\Delta_{n}^{t}} & =\frac{\left|f_{i}\left(b_{n}^{\prime}\right)-f_{i}\left(b_{n}\right)\right|}{\left(b_{n}^{\prime}-b_{n}\right)^{k}} \cdot \frac{\left|g\left(\Delta_{n} /\left(b_{n}^{\prime}-b_{n}\right)\right)\right|}{\Delta_{n} /\left(b_{n}^{\prime}-b_{n}\right)} \cdot \frac{\Delta_{n}^{1-t}}{\left(b_{n}^{\prime}-b_{n}\right)^{1-k}} \\
& \leqslant T^{k} \max (D g)\left|\frac{\Delta_{n}}{b_{n}^{\prime}-b_{n}}\right|^{1-t}\left(b_{n}^{\prime}-b_{n}\right)^{k-t},
\end{aligned}
$$

where $k-t>0,1-t>0$, and $\Delta_{n} \leqslant b_{n}^{\prime}-b_{n}$. 
Turning back to (2.12), we see that

$$
\left|f_{i}^{(t)}(b)\right| \leqslant \lim _{h \rightarrow 0}\left(T^{k}\left|b_{n}-b\right|^{k-t}+T^{k} \max (D g)\left(\frac{\Delta_{n}}{b_{n}^{\prime}-b_{n}}\right)^{1-t}\left(b_{n}^{\prime}-b_{n}\right)^{k-t}\right)=0
$$

because either $\Delta_{n} /\left(b_{n}^{\prime}-b_{n}\right)$ or $b_{n}^{\prime}-b_{n}$ tends to 0 as $h$ tends to 0 . Let $U_{b}$ be a compact neighborhood of $b$; then for the $M$ required by Definition 2.1 , we can take the number

$$
\begin{aligned}
& \max \left\{T^{k}\left|b_{n}-b\right|^{k-t}+T^{k} \max (D g)\left(b_{n}^{\prime}-b_{n}\right)^{k-t}: b_{n}, b_{n}^{\prime}, b \in U_{b}\right\} \\
& \leqslant T^{k}\left(\operatorname{diam}\left(U_{b}\right)\right)^{k-t}(1+\max (D g)) .
\end{aligned}
$$

Case 2. If $k>1$ for every $t \in \mathbb{R}, 1 \leqslant t<k$, we can suppose by induction that

$$
f_{i}^{(\tilde{t})}\left\lceil\bar{B} \equiv 0, \quad \tilde{t}= \begin{cases}{[t]} & \text { if } t \notin \mathbb{N} \\ t-1 & \text { if } t \in \mathbb{N} .\end{cases}\right.
$$

Then for all $b \in \bar{B}$,

$$
\left|f_{i}^{(t)}(b)\right|=\lim _{h \rightarrow 0} \frac{\left|f_{i}^{(\tilde{t})}(b+h)-f_{i}^{(\tilde{t})}(b)\right|}{h^{t-\tilde{t}}}
$$

and using (2.11),

$$
\begin{aligned}
\frac{\left|f_{i}^{(\tilde{t})}(b+h)-f_{i}^{(\tilde{t})}(b)\right|}{h^{t-\tilde{t}}} & =\frac{\left|f_{i}^{(\tilde{t})}(b+h)\right|}{\left(\left|b_{n}-b\right|+\Delta_{n}\right)^{t-\tilde{t}}} \\
& \leqslant \frac{\left|f_{i}^{(\tilde{t})}(b+h)-f_{i}^{(\tilde{t})}\left(b_{n}\right)\right|}{\Delta_{n}^{t-\tilde{t}}} \\
& =\left|f_{i}^{(\tilde{t}+1)}(\xi) \cdot \Delta_{n}^{1+\tilde{t}-t}\right|
\end{aligned}
$$

for some $\xi \in\left(b_{n}, b_{n}^{\prime}\right)$ (note that $f_{i}^{(\tilde{t})}(b)=f_{i}^{(\tilde{t})}\left(b_{n}\right)=0$ because $b, b_{n} \in \bar{B}$, and also that $f_{i} \in C^{\infty}$ on $\left.\left(b_{n}, b_{n}^{\prime}\right)\right)$.

From (2.7), it follows that

$$
\begin{aligned}
\left|f_{i}^{(\tilde{t}+1)}(\xi)\right| & =\frac{\left|f_{i}\left(b_{n}\right)-f_{i}\left(b_{n}^{\prime}\right)\right|}{\left(b_{n}^{\prime}-b_{n}\right)^{\tilde{t}+1}} \cdot\left|g^{(\tilde{t}+1)}\left(\frac{\xi-b_{n}}{b_{n}^{\prime}-b_{n}}\right)\right| \\
& \leqslant \frac{T^{k} \cdot\left|b_{n}-b_{n}^{\prime}\right|^{k}}{\left(b_{n}^{\prime}-b_{n}\right)^{\tilde{t}+1}} \cdot r_{\tilde{t}+1}=\frac{T^{k}\left(b_{n}^{\prime}-b_{n}\right)^{k}}{\left(b_{n}^{\prime}-b_{n}\right)^{\tilde{t}+1}} \cdot r_{\tilde{t}+1},
\end{aligned}
$$

where $r_{\tilde{t}+1}=\max \left\{g^{(\tilde{t}+1)}(\alpha) ; \alpha \in[0,1]\right\}$. 
Then for $f_{i}^{(t)}(b)$, we can write

$$
\left|f_{i}^{(t)}(b)\right| \leqslant T^{k} \cdot \frac{\Delta_{n}^{1+\tilde{t}-t} r_{\tilde{t}+1}}{\left(b_{n}^{\prime}-b_{n}\right)^{1+\tilde{t}-k}}=T^{k} r_{\tilde{t}+1} \cdot\left|\frac{\Delta_{n}}{b_{n}^{\prime}-b_{n}}\right|^{1+\tilde{t}-t} \cdot\left(b_{n}^{\prime}-b_{n}\right)^{k-t}
$$

it means that

$$
\left|f_{i}^{(t)}(b)\right| \leqslant \lim _{h \rightarrow 0}\left(T^{k} r_{\tilde{t}+1} \cdot\left|\frac{\Delta_{n}}{b_{n}^{\prime}-b_{n}}\right|^{1+\tilde{t}-t} \cdot\left(b_{n}^{\prime}-b_{n}\right)^{k-t}\right)
$$

where $k>t, \Delta_{n} /\left(b_{n}^{\prime}-b_{n}\right) \leqslant 1,1+\tilde{t} \geqslant t$. The limit is equal to 0 because either $\Delta_{n} /\left(b_{n}^{\prime}-\right.$ $\left.b_{n}\right)$ or $\left(b_{n}^{\prime}-b_{n}\right)$ tends to 0 as $h$ tends to 0 . Let $U_{b}$ be a compact neighborhood of $b$; then for the $K$ required by Definition 2.1 , we can take the number $T^{k} r_{\tilde{t}+1} \cdot\left(\operatorname{diam}\left(U_{b}\right)\right)^{k-t}$ so that, by finishing the proof of (2.9), we finish the proof of the " $C^{<k}$-extension on $\mathbb{R}$ property."

Lemma 2.11. Let $n, p \in \mathbb{N}, p \leqslant n, k \in \mathbb{R}, k \geqslant 1$. Then there exists a continuous space-filling function

$$
\pi_{k, p}^{n}=\left(\pi_{1}, \pi_{2}\right):[0,1] \stackrel{\text { onto }}{\longrightarrow}[0,1]^{n}
$$

such that $\pi_{1}:[0,1] \stackrel{D^{(p k+n-p) / k}}{\longrightarrow}[0,1]^{p}$ and $\pi_{2}:[0,1] \stackrel{D^{p k+n-p}}{\longrightarrow}[0,1]^{n-p}$ are component functions of $\pi_{k, p}^{n}$.

Proof. We consider the following:

(a) functions $\tilde{n}, \tilde{m}: \mathbb{N} \rightarrow \mathbb{N}$ such that for every $s \in \mathbb{N}$,

$$
\begin{gathered}
\tilde{n}(s)=p \cdot([k s]-[k(s-1)]), \\
\tilde{m}(s)=n-p,
\end{gathered}
$$

where $[k s]$ is the integer part of $k s$;

(b) a function $H_{\tilde{n}, \tilde{m}}:[0,1] \stackrel{\text { onto }}{\longrightarrow}[0,1]^{2}$ defined in Lemma 2.7 and let $h_{1}, h_{2}:[0$, $1] \rightarrow[0,1]$ be the component functions of $H_{\tilde{n}, \tilde{m}}$ so that for all $t \in[0,1], H_{\tilde{n}, \tilde{m}}(t)=$ $\left(h_{1}(t), h_{2}(t)\right) \in[0,1]^{2}$

(c) A function $\pi_{p, k}^{n}=\left(\pi_{1}, \pi_{2}\right):[0,1] \stackrel{\text { onto }}{\longrightarrow}[0,1]^{n}$, where

$$
\pi_{1}=f_{p} \circ h_{1}, \quad \pi_{2}=f_{n-p} \circ h_{2}
$$

Additionally,

$$
f_{p}:[0,1] \stackrel{\text { onto }}{\longrightarrow}[0,1]^{p}, \quad f_{n-p}:[0,1] \stackrel{\text { onto }}{\longrightarrow}[0,1]^{n-p}
$$

are some continuous space-filling cubes-preserving functions, the existence of which follows from Theorem 2.9. 
We establish some properties of the functions $\pi_{1}, \pi_{2}$, which we will need to finish the proof of Lemma 2.11 .

(I) If $\alpha \in K_{p[k s]+(n-p) s}^{1}$ for some $s \in \mathbb{N}$, then

$$
\begin{aligned}
f_{p}\left(h_{1}(\alpha)\right) & =\left(\pi_{1}(\alpha)\right) \in K_{[k s]}^{p}, \\
f_{n-p}\left(h_{2}(\alpha)\right) & =\left(\pi_{2}(\alpha)\right) \in K_{s}^{n-p} .
\end{aligned}
$$

We prove this property as follows. As $p[k s]+(n-p) s=\sum_{i=1}^{s} \tilde{n}(i)+\tilde{m}(i)$, by property (1) of Lemma 2.7, one has that $H_{\tilde{n}, \tilde{m}}(\alpha)=\alpha^{\prime} \times \alpha^{\prime \prime}$, where $h_{1}(\alpha)=\alpha^{\prime} \in K_{\sum_{i=1}^{s} \tilde{n}(i)}^{1}=$ $K_{p[k s]}^{1}$ and $h_{2}(\alpha)=\alpha^{\prime \prime} \in K_{\sum_{i=1}^{s} \tilde{m}(i)}^{1}=K_{(n-p) s}^{1}$. Then according to Definition 2.8 of cubespreserving functions $f_{p}, f_{n-p}$, we can see that

$$
f_{p}\left(h_{1}(\alpha)\right) \in K_{[k s]}^{p}, \quad f_{n-p}\left(h_{2}(\alpha)\right) \in K_{s}^{n-p} .
$$

(II) If $\alpha \in K_{p[k s]+(n-p) s}^{1}$ for some $s \in \mathbb{N}$, then

$$
|\alpha|=\left(S\left(\pi_{1}(\alpha)\right)\right)^{(p[k s]+(n-p) s) /[k s]}=\left(S\left(\pi_{2}(\alpha)\right)\right)^{(p[k s]+(n-p) s) / s},
$$

where $S\left(\pi_{1}(\alpha)\right), S\left(\pi_{2}(\alpha)\right)$ are lengths of sides of cubes $\pi_{1}(\alpha), \pi_{2}(\alpha)$, respectively.

We prove this property as follows. If $\alpha \in K_{p[k s]+(n-p) s}^{1}$ for some $s \in \mathbb{N}$, then by property (2.27), it means that

$$
S\left(\pi_{1}(\alpha)\right)=\frac{1}{2^{[k s]}}, \quad S\left(\pi_{2}(\alpha)\right)=\frac{1}{2^{s}} .
$$

On the other hand, $\alpha \in K_{p[k s]+(n-p) s}^{1}$ so that

$$
\begin{aligned}
|\alpha| & =\frac{1}{2^{p[k s]+(n-p) s}}, \\
|\alpha| & =\left(S\left(\pi_{1}(\alpha)\right)\right)^{(p[k s]+(n-p) s) /[k s]} \\
& =\left(S\left(\pi_{2}(\alpha)\right)\right)^{(p[k s]+(n-p) s) / s} .
\end{aligned}
$$

(III) To prove that $\pi_{1} \in D^{(p k+n-p) / k}, \pi_{2} \in D^{p k+n-p}$, it suffices to show that there exists $K>0$ such that for all $a, b \in[0,1], a<b$,

$$
\left(\operatorname{diam}\left(\pi_{1}([a, b])\right)\right)^{p+(n-p) / k}<K(b-a)>\left(\operatorname{diam}\left(\pi_{2}([a, b])\right)\right)^{p k+n-p} .
$$

We prove this property as follows. If $[a, b] \subseteq[0,1]$, then there exists $s_{1} \in \mathbb{N}, s_{1} \geqslant s_{0}$, such that

$$
\frac{1}{2^{p\left[k\left(s_{1}+1\right)\right]+(n-p)\left(s_{1}+1\right)}} \leqslant b-a \leqslant \frac{1}{2^{p\left[k s_{1}\right]+(n-p) s_{1}}} ;
$$

then $[a, b] \subseteq \alpha^{\prime} \cup \alpha^{\prime \prime}$ for some $\alpha^{\prime}, \alpha^{\prime \prime} \in K_{p\left[k s_{1}\right]+(n-p) s_{1}}^{1}, \alpha^{\prime} \cap \alpha^{\prime \prime} \neq \varnothing$, and also

$$
b-a \geqslant \frac{\left|\alpha^{\prime}\right|}{2^{p\left(\left[k\left(s_{1}+1\right)\right]-\left[k s_{1}\right]\right)+(n-p)}}>\frac{\left|\alpha^{\prime}\right|}{2^{2 k p+n}} .
$$


From property (2.29), we can see that

$$
\left|\alpha^{\prime}\right|=S\left(\pi_{1}\left(\alpha^{\prime}\right)\right)^{\left(p\left[k s_{1}\right]+(n-p) s_{1}\right) /\left[k s_{1}\right]}=S\left(\pi_{2}\left(\alpha^{\prime}\right)\right)^{\left(p\left[k s_{1}\right]+(n-p) s_{1}\right) / s_{1}}
$$

and using the fact that

$$
\begin{aligned}
& \operatorname{diam}\left(\pi_{1}\left(\alpha^{\prime} \cup \alpha^{\prime \prime}\right)\right) \leqslant 2 \sqrt{p} S\left(\pi_{1}\left(\alpha^{\prime}\right)\right), \\
& \operatorname{diam}\left(\pi_{2}\left(\alpha^{\prime} \cup \alpha^{\prime \prime}\right)\right) \leqslant 2 \sqrt{n-p} S\left(\pi_{2}\left(\alpha^{\prime}\right)\right),
\end{aligned}
$$

we get

$$
\begin{aligned}
& \operatorname{diam}\left(\pi_{1}\left(\alpha^{\prime} \cup \alpha^{\prime \prime}\right)\right) \leqslant 2 \sqrt{p}\left(2^{p\left(\left[k\left(s_{1}+1\right)\right]-\left[k s_{1}\right]\right)+n-p} \cdot(b-a)\right)^{\left[k s_{1}\right] /\left(p\left[k s_{1}\right]+(n-p) s_{1}\right)}, \\
& \operatorname{diam}\left(\pi_{2}\left(\alpha^{\prime} \cup \alpha^{\prime \prime}\right)\right) \leqslant 2 \sqrt{n-p}\left(2^{p\left(\left[k\left(s_{1}+1\right)\right]-\left[k s_{1}\right]\right)+n-p} \cdot(b-a)\right)^{s_{1} /\left(p\left[k s_{1}\right]+(n-p) s_{1}\right)} .
\end{aligned}
$$

Considering inequality (2.38), we may suppose that $\operatorname{diam}\left(\pi_{2}([a, b])\right) \leqslant 1$; also using

$$
\begin{gathered}
{[a, b] \subseteq \alpha^{\prime} \cup \alpha^{\prime \prime},} \\
{\left[k\left(s_{1}+1\right)\right]-\left[k s_{1}\right]<2 k+1,}
\end{gathered}
$$

and after the routine arithmetic transformation, we find that there exists $n_{2} \in \mathbb{N}$, which does not depend on $s_{1}$, such that

$$
\left(\operatorname{diam}\left(\pi_{2}([a, b])\right)\right)^{p k+n-p} \leqslant n_{2}(b-a) .
$$

Now we look at inequality (2.37). Knowing that $(b-a) \geqslant 1 / 2^{p\left[k\left(s_{1}+1\right)\right]+(n-p)\left(s_{1}+1\right)}$, inequality (2.37) can be transformed into

$$
\begin{aligned}
\operatorname{diam}\left(\pi_{1}([a, b])\right) \leqslant & 2 \sqrt{p}\left(2^{2 k p+n} \cdot(b-a)\right)^{1 /(p+(n-p) / k)} \\
& \times\left(2^{2 k p+n} \cdot \frac{1}{2^{p\left[k\left(s_{1}+1\right)\right]+(n-p)\left(s_{1}+1\right)}}\right)^{\left[k s_{1}\right] /\left(p\left[k s_{1}\right]+(n-p) s_{1}\right)-1 /(p+(n-p) / k)} .
\end{aligned}
$$

Note that $\left[k s_{1}\right] /\left(p\left[k s_{1}\right]+(n-p) s_{1}\right)-1 /(p+(n-p) / k) \leqslant 0$ and there exists a number $n_{1} \in \mathbb{N}$, which does not depend on $s_{1}$, such that

$$
\left(\operatorname{diam}\left(\pi_{1}([a, b])\right)\right)^{p+(n-p) / k} \leqslant n_{1}(b-a)
$$

The existence of such $n_{1}$ only depends on whether the expression

$$
-\left(p\left[k\left(s_{1}+1\right)\right]+(n-p)\left(s_{1}+1\right)\right)\left(\frac{\left[k s_{1}\right]}{p\left[k s_{1}\right]+(n-p) s_{1}}-\frac{1}{p+(n-p) / k}\right)
$$


is bounded above. Expression (2.43) can be easily transformed into

$$
\frac{p\left[k\left(s_{1}+1\right)\right]+(n-p)\left(s_{1}+1\right)}{p\left[k s_{1}\right]+(n-p) s_{1}} \times \frac{(n-p)\left(k s_{1}-\left[k s_{1}\right]\right)}{p k+n-p} ;
$$

noticing that the first multiple is bounded and $k s_{1}-\left[k s_{1}\right]<1$, it follows that expression (2.43) is bounded above.

Now choosing $K=\max \left\{n_{1}, n_{2}\right\}$, we finish the proof of property (2.32).

(IV) $\pi_{k, p}^{n}$ is continuous space-filling function.

This is proved as follows. (1) Continuity of $\pi_{k, p}^{n}$ follows from the continuity of the component functions $\pi_{1}, \pi_{2}$ that are continuous as compositions of the continuous functions $f_{p}$ with $h_{1}$ and $f_{n-p}$ with $h_{2}$, respectively.

(2) Now let $y=\left(y_{1}, y_{2}\right) \in[0,1]^{n}$, where $y_{1} \in[0,1]^{p}, y_{2} \in[0,1]^{n-p}$.

Functions $f_{p}:[0,1] \rightarrow[0,1]^{p}$ and $f_{n-p}:[0,1] \rightarrow[0,1]^{n-p}$ are space filling so that there exist $z_{1}, z_{2} \in[0,1]$ such that $f_{p}\left(z_{1}\right)=y_{1}, f_{n-p}\left(z_{2}\right)=y_{2}$. On the other hand, the point $\left(z_{1}, z_{2}\right) \in[0,1]^{2}$ and $H_{\tilde{n}, \tilde{m}}:[0,1] \stackrel{\text { onto }}{\longrightarrow}[0,1]^{2}$ so that there exists $t \in[0,1]: H_{\tilde{n}, \tilde{m}}(t)=\left(z_{1}, z_{2}\right)$ or $h_{1}(t)=z_{1}, h_{2}(t)=z_{2}$, and by the definition of $\pi_{k, p}^{n}: \pi_{k, p}^{n}(t)=y$.

From (1) and (2), it follows that $\pi_{k, p}^{n}$ is a continuous space-filling function.

LemmA 2.12. If $f \in C^{k \cdot \lambda}\left(\mathbb{R}^{n}, \mathbb{R}^{n}\right), k \geqslant 1, \lambda \in[0,1)$, and $D f_{x}$ is a linear isomorphism, then $f$ is invertible in a neighborhood of $x$ and $f^{-1}$ is of class $C^{k \cdot \lambda}$.

Proof. Similar to the proof of the $C^{k+\beta+}$ inverse function theorem in [7].

Lemma 2.13. If $k \geqslant 1, f \in C^{k \cdot \lambda}\left(\mathbb{R}^{n}, R\right), x \in \mathbb{R}^{n}, f(x)=0, D f(x) \neq 0$, then there is a neighborhood $N$ of $x$ in $\mathbb{R}^{n}$ and $C^{k \cdot \lambda}(n-1)$-submanifold $S \subseteq \mathbb{R}^{n}$ such that $f^{-1}(0) \cap N \subset S$.

Proof. Similar to the proof of Zygmund preimage theorem in [8].

Lemmas 2.14 and 2.15 are generalized Morse vanishing lemma and Morse theorem; see Morse [5], and for more general version of the lemmas, see also Norton [7, 8] and Moreira [4].

Lemma 2.14. Let $k$, $n$ be nonnegative integers, $\lambda \in[0,1)$, and $A \subseteq \mathbb{R}^{n}=\mathbb{R}^{n-p} \times \mathbb{R}^{p}$ for some $p \leqslant n$. Then there are sets $A_{1}, A_{2}, \ldots \subseteq A$ such that $A=\bigcup_{i=1}^{\infty} A_{i}$, where for each $i=1,2, \ldots$, there is a function $\psi_{i}: V_{i} \times B_{i} \stackrel{C^{1}}{\longrightarrow} \mathbb{R}^{n}, V_{i}$ is a bounded ball in $\mathbb{R}^{p}$ and $B_{i}$ is a bounded ball in some $\mathbb{R}^{r_{i}}\left(0 \leqslant r_{i} \leqslant n-p\right)$ such that

$$
\begin{gathered}
\psi_{i}(x, y)=\left(x, \tilde{\psi}_{i}(x, y)\right) \\
\left|\psi_{i}\left(x_{1}, y_{1}\right)-\psi_{i}\left(x_{2}, y_{2}\right)\right| \geqslant\left|\left(x_{1}, y_{1}\right)-\left(x_{2}, y_{2}\right)\right| \quad \forall\left(x_{1}, y_{1}\right),\left(x_{2}, y_{2}\right) \in V_{i} \times B_{i}, \\
A_{i} \subset \psi_{i}\left(V_{i} \times B_{i}\right)
\end{gathered}
$$

with the following property: every $f \in C^{k \cdot \lambda}\left(\mathbb{R}^{n}, \mathbb{R}\right)$ vanishing on $A$ satisfies for each $i$ and some $K_{i} \geqslant 0$,

$$
\begin{aligned}
& \left|f\left(\psi_{i}\left(x_{0}, y\right)\right)-f\left(\psi_{i}\left(x_{0}, y_{0}\right)\right)\right| \\
& \quad \leqslant K_{i}\left|y-y_{0}\right|^{k+\lambda} \quad \forall\left(x_{0}, y\right) \in V_{i} \times B_{i}, \psi_{i}\left(x_{0}, y_{0}\right) \in A_{i} .
\end{aligned}
$$


Proof. Fix $\lambda$. The proof is by double induction on $n$ and $k$. Let $\langle n, k\rangle$ stand for the statement of the lemma for $\mathbb{R}^{n}$ and $C^{k \cdot \lambda}$. We will prove $\langle 0, k\rangle$ for all $k,\langle n, 0\rangle$ for all $n$, and $\langle n-1, k\rangle$ and $\langle n, k-1\rangle$ imply $\langle n, k\rangle$.

(a) Proof of $\langle 0, k\rangle$ for all $k$ is trivial.

(b) Proof of $\langle n, 0\rangle$ for all $n$ follows directly from the definition of $f \in C^{0 \cdot \lambda}$.

(c) Induction step: we assume $\langle n-1, k\rangle$ and $\langle n, k-1\rangle$, and we prove $\langle n, k\rangle$.

Define

$$
\begin{aligned}
A^{* *}= & \left\{(x, y) \in A: x \in \mathbb{R}^{p}, y \in \mathbb{R}^{n-p}, \text { and every } g \in C^{k \cdot \lambda}\left(\mathbb{R}^{n}, \mathbb{R}\right)\right. \\
& \left.\quad \text { vanishing on } A \text { satisfies } D_{y} g \equiv 0 \text { on } A\right\}, \\
A^{*}= & A \backslash A^{* *} .
\end{aligned}
$$

We prove the result separately for $A^{* *}$ and $A^{*}$.

On $A^{* *}$. Since $f$ vanishes on $A, D_{y} f=\left(D_{y_{j}} f\right)_{p<j \leqslant n} \equiv 0$ on $A^{* *}$, where $y=\left(y_{p+1}, \ldots\right.$, $\left.y_{j}, \ldots, y_{n}\right)$ so that for each $j(p<j \leqslant n)$, if any, $D_{y_{j}} f$ vanishes on $A^{* *}$, and $D_{y_{j}} f \in$ $C^{k-1 \cdot \lambda}\left(\mathbb{R}^{n}, \mathbb{R}\right)$. Hence by the $\langle n, k-1\rangle$ hypothesis, we have $A^{* *}=\bigcup_{i=1}^{\infty} A_{i}^{* *}, A_{i}^{* *} \subset$ $\psi_{i}\left(V_{i} \times B_{i}\right), \psi_{i}$ as in the statement, and

$$
\begin{gathered}
\left(x_{0}, y\right),\left(x_{0}, y_{0}\right) \in V_{i} \times B_{i}, \\
\psi_{i}\left(x_{0}, y_{0}\right) \in A_{i}^{* *} \Longrightarrow \exists K_{i j} \geqslant 0 \text { such that } \forall j(p<j \leqslant n), \\
\left|D_{y_{j}} f\left(\psi_{i}\left(x_{0}, y\right)\right)-D_{y_{j}} f\left(\psi_{i}\left(x_{0}, y_{0}\right)\right)\right| \leqslant K_{i j}\left|y-y_{0}\right|^{k-1+\lambda},
\end{gathered}
$$

or let $K_{i}=\sqrt{n-p} \max _{p<j \leqslant n} K_{i j}$, then

$$
\begin{gathered}
\left(x_{0}, y\right),\left(x_{0}, y_{0}\right) \in V_{i} \times B_{i}, \\
\psi_{i}\left(x_{0}, y_{0}\right) \in A_{i}^{* *} \Longrightarrow\left|D_{y} f\left(\psi_{i}\left(x_{0}, y\right)\right)-D_{y} f\left(\psi_{i}\left(x_{0}, y_{0}\right)\right)\right| \leqslant K_{i}\left|y-y_{0}\right|^{k-1+\lambda} .
\end{gathered}
$$

Now by the mean value theorem,

$$
\begin{gathered}
\left(x_{0}, y\right),\left(x_{0}, y_{0}\right) \in V_{i} \times B_{i}, \\
\psi_{i}\left(x_{0}, y_{0}\right) \in A_{i}^{* *} \Longrightarrow \\
=D\left(f \circ \psi_{i}\left(x_{0}, y\right)\right)-f\left(\psi_{i}\left(x_{0}, y_{0}\right)\right) \\
\quad\left(\text { for some } \theta \in B_{0} \text { lying on a line segment between } y \text { and } y_{0}\right) \\
=\left(\left(x_{0}, y\right)-\left(x_{0}, y_{0}\right)\right) \\
\left.=D f\left[\psi_{i}\left(x_{0}, \theta\right)\right] \cdot D \psi_{i}\left(x_{0}, \theta\right)\right) \cdot\left(0, y-y_{0}\right) \\
\end{gathered}
$$

We recall that $\psi_{i}(x, y)=\left(x, \tilde{\psi}_{i}(x, y)\right)$ so that $D \psi_{i}(x, y)$ is presented in the following matrix consisting of $n$ rows, where the last $n-p$ rows constitute the Jacobian matrix for 
the function $\tilde{\psi}_{i}(x, y)$ :

$$
\left[\begin{array}{llll}
1 & \text { Zeros } & & \\
0 & 1 & \text { Zeros } & \\
& \cdots & \cdots & \text { Zeros } \\
\text { Zeros } & & 1 & \\
& \multicolumn{3}{c}{D \tilde{\psi}_{i}(x, y)}
\end{array}\right]
$$

Thus

$$
D \psi_{i}\left(x_{0}, \theta\right) \cdot\left(0, y-y_{0}\right)=\left(0, D \tilde{\psi}_{i}\left(x_{0}, \theta\right) \cdot\left(0, y-y_{0}\right)\right)
$$

Knowing that $(a, b) \cdot(0, c)=(0, b) \cdot(0, c)$, we get

$$
\begin{aligned}
& D f\left[\psi_{i}\left(x_{0}, \theta\right)\right] \cdot\left(0, D \tilde{\psi}_{i}\left(x_{0}, \theta\right) \cdot\left(0, y-y_{0}\right)\right) \\
& \quad=\left(0, D_{y} f\left[\psi_{i}\left(x_{0}, \theta\right)\right]\right) \cdot\left(0, D \tilde{\psi}_{i}\left(x_{0}, \theta\right) \cdot\left(0, y-y_{0}\right)\right) .
\end{aligned}
$$

Now using (2.52), (2.53), (2.49) in (2.50), we have

$$
\begin{aligned}
& \left|f\left(\psi_{i}\left(x_{0}, y\right)\right)-f\left(\psi_{i}\left(x_{0}, y_{0}\right)\right)\right| \\
& \leqslant\left|D_{y} f\left[\psi_{i}\left(x_{0}, \theta\right)\right]\right| \cdot\left|0, D \tilde{\psi}_{i}\left(x_{0}, \theta\right) \cdot\left(0, y-y_{0}\right)\right| \\
& \leqslant\left|D_{y} f\left[\psi_{i}\left(x_{0}, \theta\right)\right]-D_{y} f\left(\psi_{i}\left(x_{0}, y_{0}\right)\right)\right| \tilde{K}_{i}\left|\left(0, y-y_{0}\right)\right| \\
& \quad\left(\text { where } D_{y} f\left(\psi_{i}\left(x_{0}, y_{0}\right)\right)=0 \text { because } \psi_{i}\left(x_{0}, y_{0}\right) \in A^{* *},\right. \\
& \text { and } \tilde{K}_{i} \text { is a Lipschitz constant of the } C^{1} \text {-function } \tilde{\psi}_{i} \\
& \left.\quad \text { on the bounded cube } V_{i} \times B_{i}, \text { that we may suppose to exist }\right) \\
& \leqslant K_{i}\left|\theta-y_{0}\right|^{k-1+\lambda} \tilde{K}_{i}\left|y-y_{0}\right| \leqslant K_{i} \tilde{K}_{i}\left|y-y_{0}\right|^{k+\lambda} .
\end{aligned}
$$

On $A^{*}$. If $\left(x_{0}, y_{0}\right) \in A^{*}$, there is $g$ as above, and by Lemma 2.13, there is $\varepsilon>0$ such that $g^{-1}(0) \cap B_{\varepsilon}\left(x_{0}, y_{0}\right)$ is contained in the image of $\psi: V \times B \stackrel{C^{k \cdot \lambda}}{\longrightarrow} U$, where $B$ is a ball in $\mathbb{R}^{n-p-1}, V$ is a ball in $\mathbb{R}^{p}$ as in the statement, and $A \cap B_{\varepsilon}\left(x_{0}, y_{0}\right) \subseteq g^{-1}(0)$. Taking a countable subcovering of $A^{*}$ by these balls, we reduce the proof in this case to a case with smaller $n$.

Lemma 2.15. Let $k$, $n$ be nonnegative integers, $\lambda \in[0,1)$, and $A \subseteq \mathbb{R}^{n}=\mathbb{R}^{n-p} \times \mathbb{R}^{p}$ for some $p \leqslant n$. Then there are sets $A_{1}, A_{2}, \ldots \subseteq A$ such that $A=\bigcup_{i=1}^{\infty} A_{i}$, where for each $i=1,2, \ldots$, there is a function $\psi_{i}: V_{i} \times B_{i} \stackrel{C^{1}}{\longrightarrow} \mathbb{R}^{n}, V_{i}$ is a bounded ball in $\mathbb{R}^{p}$ and $B_{i}$ is a bounded ball in some $\mathbb{R}^{r_{i}}\left(0 \leqslant r_{i} \leqslant n-p\right)$ such that (2.45) holds with the following property: every $f \in$ $C^{k \cdot \lambda}\left(\mathbb{R}^{n}, \mathbb{R}\right)$, such that $D_{y} f \equiv 0$ in $A$, satisfies (2.46) for each $i$, and some $K_{i} \geqslant 0$. 
Proof. The same as in the case "On $A^{* *}$ " of Lemma 2.14 if we make there the following corrections:

(1) delete " $f$ vanishes on $A$,"

(2) replace " $\langle n, k-1\rangle$ hypothesis" with "Lemma 2.14,"

(3) replace $A^{* *}$ with $A$,

(4) replace $A_{i}^{* *}$ with $A_{i}$.

\section{Proof of the main theorem}

It follows from [2, Theorem 1] and Theorems 3.2, 3.3, and 3.4.

Definition 3.1. A set $A \subseteq \mathbb{R}^{m}$ is a $Z_{k}$-set for some positive $k \in \mathbb{R}$ if $A$ is a subset of $\Phi([0,1])$ for some continuous function $\Phi:[0,1] \rightarrow \mathbb{R}^{m}$ such that there exists $P>0$ such that for all $a \in \Phi^{-1}(A), b \in[0,1]$, the following is true:

$$
|\Phi(a)-\Phi(b)| \leqslant P|a-b|^{k}
$$

If a set $A=\bigcup_{i \in \mathbb{N}} A_{i}$ and every $A_{i}$ is a $Z_{k}$-set for some fixed $k$, then the set $A$ is called a $\sigma-Z_{k}$-set.

Theorem 3.2. Let $F: \mathbb{R}^{n} \stackrel{C^{k \cdot \lambda}}{\longrightarrow} \mathbb{R}^{m}, k \in \mathbb{N}, \lambda \in[0,1)$. Then $F\left(C_{p}(F)\right)$ is $\sigma-$ $Z_{1 /(p+(n-p) /(k+\lambda))}$-set in $\mathbb{R}^{m}$ for every $p<\min \{m, n\}$.

Proof. Since $C_{p}(F)=\bigcup_{r=0}^{p}\left\{x \in \mathbb{R}^{n}: \operatorname{rank}(D F(x))=r\right\}$ and $r+(n-r) /(k+\lambda) \leqslant p+(n-$ $p) /(k+\lambda)$ for $0 \leqslant r \leqslant p$, we may restrict our attention to $\tilde{C}_{p}(F)=\{x \in \mathbb{R}: \operatorname{rank}(D F(x))=$ $p\}$.

If $x_{0} \in \tilde{C_{p}}(F)$, we can consider with accuracy to within a change of coordinates of class $C^{k \cdot \lambda}$ that

$$
\begin{aligned}
& F(z, y)=(z, G(z, y)), \quad(z, y) \in \mathbb{R}^{p} \times \mathbb{R}^{n-p}, \\
& G(z, y) \in \mathbb{R}^{m-p} \quad \text { in a neighborhood } U \text { of } x_{0}=\left(z_{0}, y_{0}\right) .
\end{aligned}
$$

We have $x=(z, y) \in \tilde{C}_{p}(F)$ if and only if $D_{y} G(z, y)=0$. By applying the results of Lemma 2.15 to a set $A=\left\{(z, y) \in U: D_{y} G(z, y)=0\right\}$, we obtain the decomposition $A=$ $\bigcup_{i=1}^{\infty} A_{i}, A_{i} \subset \psi_{i}\left(V_{i} \times B_{i}\right)$. It is not difficult to see that the proof of the theorem is reducible to a proof of the following statement: for each $i$, and some $K_{i} \geqslant 0$,

$$
F\left(A_{i} \cap \psi_{i}\left(V_{i} \times B_{i}\right)\right) \text { is a } Z_{p+(n-p)(k+\lambda)} \text {-set. }
$$

Since every component function $F_{j}: \mathbb{R}^{n} \rightarrow \mathbb{R}(1 \leqslant j \leqslant m)$ of the function $F=\left(F_{1}, \ldots, F_{j}\right.$, $\left.\ldots, F_{m}\right)$ satisfies $F_{j} \in C^{k \cdot \lambda}, D_{y} F_{j} \equiv 0$ on $A$, then by Lemma 2.15, $F_{j}$ satisfies $(2.46)$, and then it is not difficult to see that $F$ itself satisfies (2.46), it means that for each $i$; and for some $K_{i} \geqslant 0$,

$$
\begin{aligned}
& \left|F\left(\psi_{i}\left(x_{0}, y\right)\right)-F\left(\psi\left(x_{0}, y_{0}\right)\right)\right| \\
& \quad \leqslant K_{i}\left|y-y_{0}\right|^{k+\lambda} \quad \forall\left(x_{0}, y\right) \in V_{i} \times B_{i},\left(x_{0}, y_{0}\right) \in A_{i} .
\end{aligned}
$$


Now we fix such $A_{i}$. We may suppose without loss of generality that $V_{i} \in K_{\left[(k+\lambda) s_{0}\right]}^{p}, B_{i} \in$ $K_{s_{0}}^{n-p}$ for some $s_{0} \in \mathbb{N}$, where $\left[(k+\lambda) s_{0}\right]$ is an integer part of $(k+\lambda) s_{0}$.

We consider the following:

(a) a set $\alpha_{0} \in K_{p\left[(k+\lambda) s_{0}\right]+(n-p) s_{0}}^{1}$;

(b) a set $D=\left\{\alpha \in K_{p[(k+\lambda) s]+(n-p) s}^{1}, \alpha \subseteq \alpha_{0}\right\}$;

(c) a function $\pi=\left(\pi^{*}, \pi^{* *}\right)$, where $\pi^{*}=I_{1} \circ \pi_{1} \uparrow \alpha_{0}, \pi^{* *}=I_{2} \circ \pi_{2}\left\lceil\alpha_{0} ; \pi_{1}, \pi_{2}\right.$ are the component functions of a function $\pi_{k+\lambda, p}^{n}$, defined in Lemma 2.11; $I_{1}$ : $\pi^{*}\left(\alpha_{0}\right) \stackrel{\text { onto }}{\longrightarrow} V_{i}, I_{2}: \pi^{* *}\left(\alpha_{0}\right) \stackrel{\text { onto }}{\longrightarrow} B_{i}$ are the identity maps;

(d) and finally a function $\Phi:[0,1] \rightarrow F\left(\psi_{i}\left(V_{i} \times B_{i}\right)\right) \subseteq \mathbb{R}^{m}$ such that

$$
\begin{aligned}
& \Phi(a)=F\left(\psi_{i}(\pi(a))\right) \quad \forall \alpha \in \alpha_{0}, \\
& \Phi(a)=\Phi(\underline{a}) \quad \forall a \leqslant \underline{a}=\min _{x \in \alpha_{0}} x, \\
& \Phi(a)=\Phi(\bar{a}) \quad \forall a \geqslant \bar{a}=\max _{x \in \alpha_{0}} x .
\end{aligned}
$$

It follows from property (3.2) of $F$ and the property of $\psi_{i}$ (see Lemma 2.15) that $\Phi(a)=$ $\left(\pi^{*}(a), G\left(\psi_{i}(\pi(a))\right)\right)$ for every $a \in[0,1]$.

Now, we are ready to prove (3.3).

From (c) and (d), we see that $F\left(A_{i} \cap \psi_{i}\left(V_{i} \times B_{i}\right)\right) \subseteq \Phi([0,1])$.

The function $\Phi:[0,1] \rightarrow \mathbb{R}^{m}$ is continuous as a composition of continuous functions (see $(\mathrm{d}))$. To finish the proof of property (3.3), we need to evaluate $\Phi([a, b])$ for any $[a, b] \subseteq \alpha_{0}$ such that $\psi_{i}(\pi(a)) \in A_{i}$ or $\psi_{i}(\pi(b)) \in A_{i}$.

We suppose that $\psi_{i}(\pi(a)) \in A_{i}$ (the other case is similar to this one). Then

$$
\begin{aligned}
\mid \Phi(b) & -\Phi(a) \mid \\
= & \left|F\left(\psi_{i}(\pi(b))\right)-F\left(\psi_{i}(\pi(a))\right)\right| \\
\leqslant & \left|F\left(\psi_{i}\left(\pi^{*}(a), \pi^{* *}(b)\right)\right)-F\left(\psi_{i}\left(\pi^{*}(a), \pi^{* *}(a)\right)\right)\right| \\
& +\left|F\left(\psi_{i}\left(\pi^{*}(b), \pi^{* *}(b)\right)\right)-F\left(\psi_{i}\left(\pi^{*}(a), \pi^{* *}(b)\right)\right)\right| \\
\leqslant & K_{i}\left|\pi^{* *}(b)-\pi^{* *}(a)\right|^{(k+\lambda)}+L\left|\pi^{*}(b)-\pi^{*}(a)\right|
\end{aligned}
$$

(by (3.4) and that $L$ is a Lipschitz constant of the $C^{1}$

function $F \circ \psi_{i} \uparrow V_{i}$ which we may suppose to exist)

$$
\leqslant K_{i}\left(K^{*}|a-b|\right)^{(k+\lambda) /(p(k+\lambda)+n-p)}+L\left(K^{* *}|a-b|\right)^{(k+\lambda) /(p(k+\lambda)+n-p)}
$$

(for some positive numbers $K^{*}, K^{* *}$ by (c) and Lemma 2.11)

$$
\leqslant P \cdot|a-b|^{(k+\lambda) /(p(k+\lambda)+n-p)},
$$

where $P=\left(K_{i}+L\right)\left(\max \left\{K^{*}, K^{* *}\right\}\right)^{(k+\lambda) /(p(k+\lambda)+n-p)}$.

We finish the proof of property (3.3) and thereby the proof of Theorem 3.2. 
Theorem 3.3. If $A \subseteq \mathbb{R}^{m}$ is a $Z_{k}$-set in $\mathbb{R}^{m}$, then $A \subseteq f\left(\Sigma_{k} f\right)$ for some $f:[a, b] \subseteq \mathbb{R}^{1} \stackrel{C^{<k}}{\longrightarrow}$ $\mathbb{R}^{m}$.

Proof. If $A \subseteq \mathbb{R}^{m}$ is a $Z_{k}$-set in $\mathbb{R}^{m}$, then by Definition 3.1, $A$ is a subset of $\Phi([0,1])$ for some continuous function $\Phi:[0,1] \rightarrow \mathbb{R}^{m}$ such that there exists $P>0$ such that for all $a \in \Phi^{-1}(A), b \in[0,1]$, the following is true:

$$
|\Phi(a)-\Phi(b)| \leqslant P|a-b|^{k}
$$

so that a function $F=\Phi\left\lceil\Phi^{-1}(A)\right.$ is a $D^{1 / k}$-function such that $A \subseteq \operatorname{range}(F)$.

Now, the conclusion of this theorem follows from the " $C^{<k}$-extension on $\mathbb{R}$ property."

Theorem 3.4. If $A=\bigcup_{i \in \mathbb{N}}, A_{i} \subseteq \mathbb{R}^{m}$, and for all $i \in \mathbb{N}$, there exist $f_{i}:\left[a_{i}, b_{i}\right] \subseteq \mathbb{R}^{1} \stackrel{C^{k(<k)}}{\longrightarrow} \mathbb{R}^{m}$ such that $A_{i} \subseteq f_{i}\left(\Sigma_{k} f_{i}\right)$. Then there exists $f: \mathbb{R}^{1} \stackrel{C^{k(k k)}}{\longrightarrow} \mathbb{R}^{m}$ such that $A \subseteq f\left(\Sigma_{k} f\right)$.

Proof. We may suppose without loss of generality that for all $i \in \mathbb{N}$,

(i) $A_{i}$ is closed,

(ii) $a_{i}, b_{i} \in \sum_{k} f_{i}$,

(iii) $\left\{\left[a_{i}, b_{i}\right], i \in \mathbb{N}\right\}$ is disjoint,

(iv) $\left|\left[b_{i}, a_{i+1}\right]\right|>\max \left\{\left|f_{i+1}(a)-f_{j}(b)\right|\right\} ; j \leqslant i, a \in\left[a_{i+1}, b_{i+1}\right], b \in \bigcup_{j=1}^{i}\left[a_{j}, b_{j}\right]$.

Using functions similar to (2.7), we can construct $C^{\infty}$-function $f_{0}: \mathbb{R}^{1} \backslash \bigcup_{i \in \mathbb{N}}\left(a_{i}, b_{i}\right) \rightarrow$ $\mathbb{R}^{m}$ such that $\left\{a_{i}, b_{i} ; i \in \mathbb{N}\right\} \subseteq \Sigma_{\infty} f_{0}$. Then define the required function $f: \mathbb{R}^{1} \rightarrow \mathbb{R}^{m}$ as follows:

$$
\begin{gathered}
f \uparrow\left(\mathbb{R}^{1} \backslash \bigcup_{i \in \mathbb{N}}\left(a_{i}, b_{i}\right)\right)=f_{0}, \\
f \uparrow\left[a_{i}, b_{i}\right]=f_{i}, \quad i \in \mathbb{N} .
\end{gathered}
$$

\section{References}

[1] A. Ainouline, Necessary and sufficient condition of the Morse-Sard theorem for real valued functions, preprint, 2003, http://arxiv.org/abs/math.CA/0405012.

[2] - Vector valued functions not constant on connected sets of critical points, preprint, 2003, http://arxiv.org/abs/math.GT/0404404.

[3] S. M. Bates and A. Norton, On sets of critical values in the real line, Duke Math. J. 83 (1996), no. 2, 399-413.

[4] C. G. T. de A. Moreira, Hausdorff measures and the Morse-Sard theorem, Publ. Mat. 45 (2001), no. $1,149-162$.

[5] A. P. Morse, The behavior of a function on its critical set, Ann. of Math. (2) 40 (1939), no. 1, 62-70.

[6] J. R. Munkres, Elementary Differential Topology, Annals of Mathematics Studies, no. 54, Princeton University Press, New Jersey, 1963.

[7] A. Norton, A critical set with nonnull image has large Hausdorff dimension, Trans. Amer. Math. Soc. 296 (1986), no. 1, 367-376.

[8] - The Zygmund Morse-Sard theorem, J. Geom. Anal. 4 (1994), no. 3, 403-424.

[9] H. Sagan, Space-Filling Curves, Universitext, Springer-Verlag, New York, 1994. 
776 Critical values lie on a line

[10] H. Whitney, A function not constant on a connected set of critical points, Duke Math. J. 1 (1935), no. 4, 514-517.

[11] Y. Yomdin, The geometry of critical and near-critical values of differentiable mappings, Math. Ann. 264 (1983), no. 4, 495-515.

Azat Ainouline: Department of Mathematics \& Statistics, University of Calgary, 2500 University Drive NW, Calgary, AB, Canada T2N $1 \mathrm{~N} 4$.

E-mail address: azat@math.ucalgary.ca 


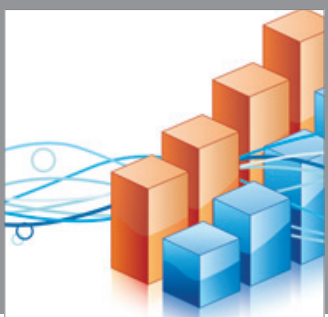

Advances in

Operations Research

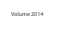

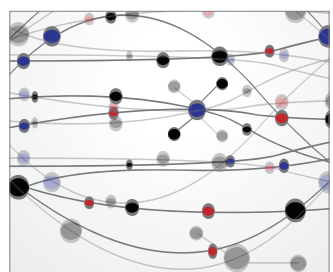

\section{The Scientific} World Journal
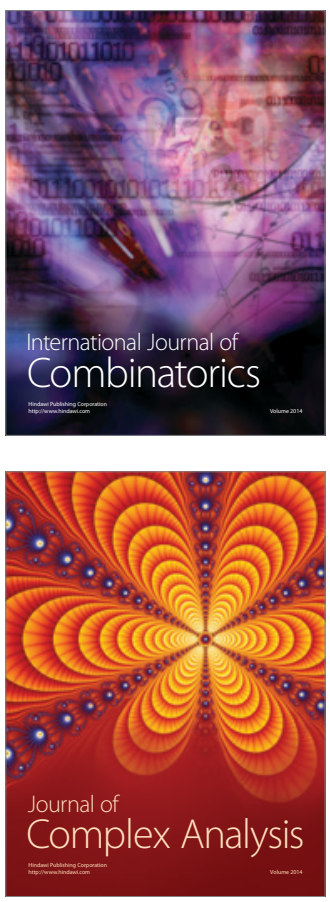

International Journal of

Mathematics and

Mathematical

Sciences
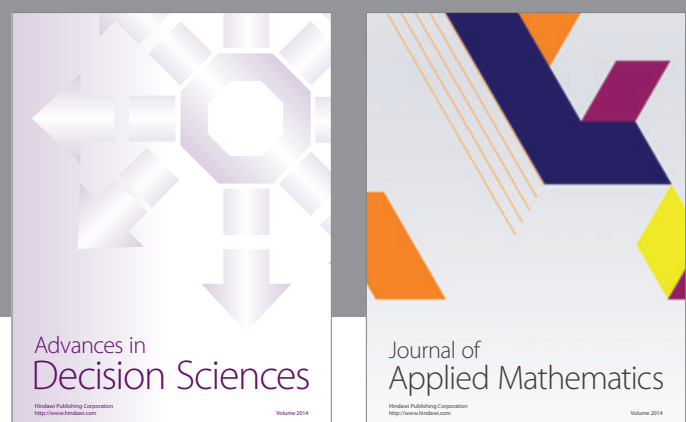

Journal of

Applied Mathematics
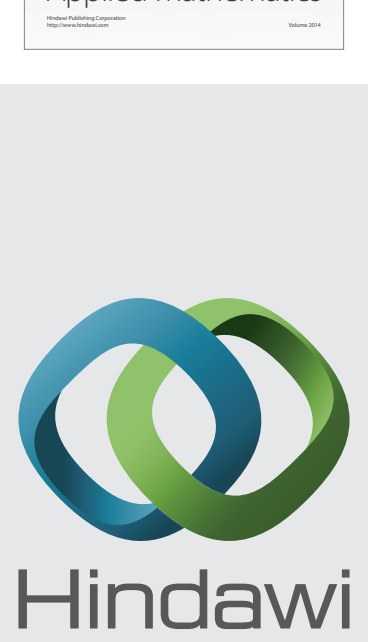

Submit your manuscripts at http://www.hindawi.com
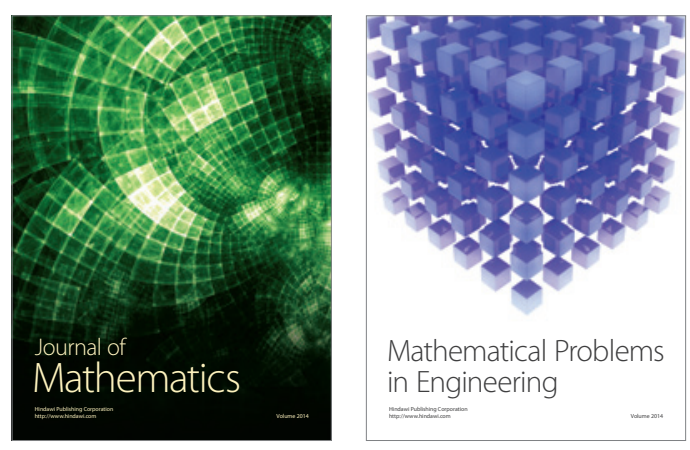

Mathematical Problems in Engineering
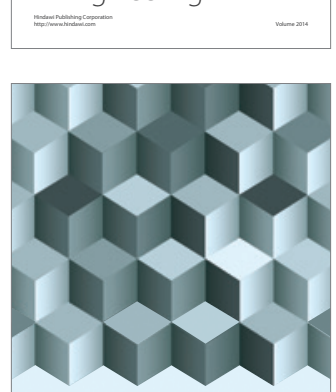

Journal of

Function Spaces
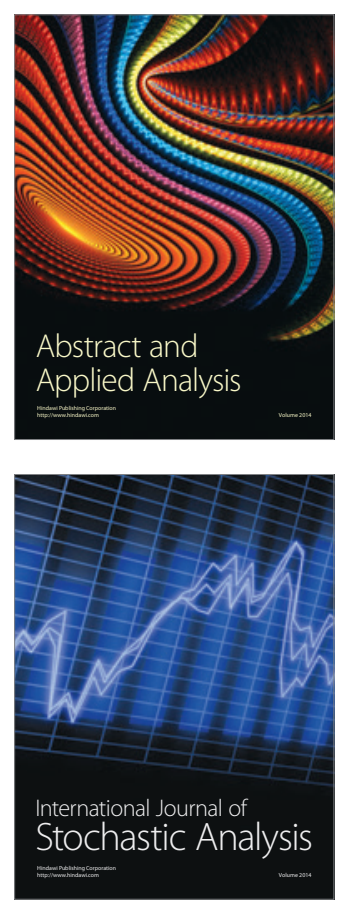

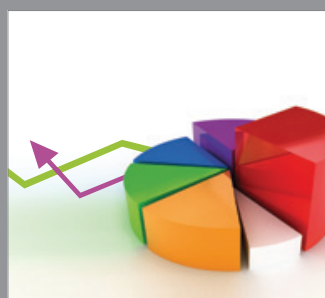

ournal of

Probability and Statistics

Promensencen
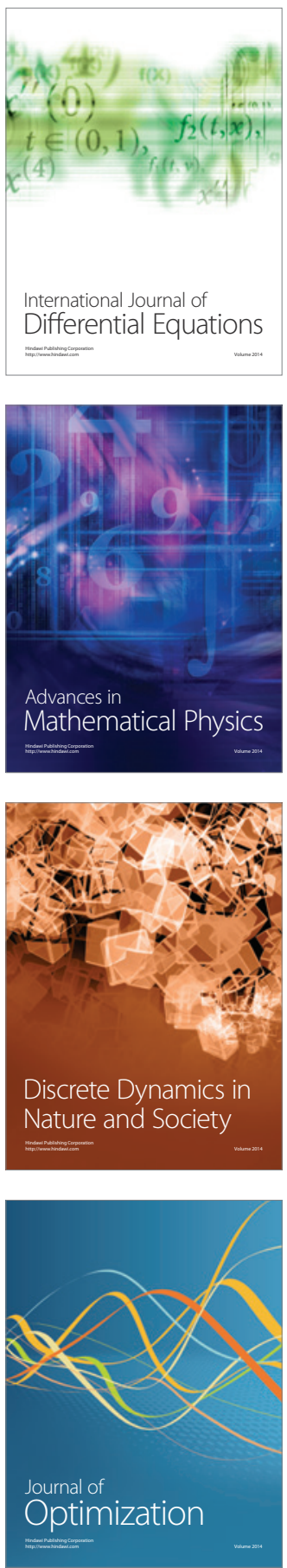